\title{
Monitoramento e Suporte a Controle de Estufas Climatizadas Utilizando Nuvem e MQTT
}

\author{
Vinícius M. da Costa ${ }^{1}$, Marcello Matheus E. Nery ${ }^{1}$, \\ Guilherme P. R. Paes ${ }^{1}$, Pedro R. F. de Souza ${ }^{1}$, \\ Eduardo K. Almentero ${ }^{1}$, Luiz M. C. Branco ${ }^{1}$ \\ ${ }^{1}$ Departamento de Computação \\ Universidade Federal Rural do Rio de Janeiro (UFRRJ) \\ Seropédica - RJ - Brasil \\ $\{$ vinimartinsc, contact.mnery, guilhermep98, pedro.raposo.dev, \\ ekalmentero, luizmaltar\}@gmail.com
}

\begin{abstract}
Agribusiness has an increasingly important share of the Brazilian GDP. Agriculture is greatly influenced by climatic conditions and the increase in global temperature enhances these adverse effects. Cultivation through greenhouses allows for management that uses a certain control of climatic conditions, aiming at increasing quality and productivity. Increasing the control of greenhouse conditions makes it possible to increase the benefits of this type of cultivation, and, in this context, technology can play a fundamental role, especially when we use the concept of IoT (Internet of Things). This work proposes an infrastructure for the automated control of greenhouses, based on the concept of IoT and technologies such as Raspberry Pi, MQTT (Message Queuing Telemetry Transport), and mobile applications.
\end{abstract}

Resumo. O agronegócio tem uma participação cada vez mais importante no PIB brasileiro. A agricultura é muito influenciada pelas condições climáticas e o aumento da temperatura global potencializa estes efeitos adversos. O cultivo através de estufas permite um manejo que usufrui de certo controle das condições climáticas, visando o aumento da qualidade e produtividade. Aumentar o controle das condições de uma estufa possibilita aumentar os benefícios deste tipo de cultivo, e, neste contexto, a tecnologia pode desempenhar um papel fundamental, principalmente quando utilizamos o conceito de IoT (Internet das coisas). Este trabalho propõe uma infraestrutura para o controle automatizado de estufas, utilizando como base o conceito de IoT e tecnologias como Raspberry Pi, MQTT (Message Queuing Telemetry Transport) e aplicativos móveis.

\section{Introdução}

De acordo com o CEPEA (Centro de Estudos Avançados em Economia Aplicada), em 2020 o agronegócio foi responsável por 26,2\% do PIB brasileiro [CEPEA, 2020]. Quando analisamos a série histórica deste, constata-se aumento de 5,4\% de 2019 para 2020, enquanto os outros setores (insumos, indústria e serviços) perderam espaço. Este é um forte indício do quanto o setor é importante para o país atualmente, e continuará sendo no futuro. A produtividade agrícola é fortemente influenciada pelo clima. É comum nos depararmos com notícias que relatam perdas grandes devido a efeitos climáticos adversos, 
como chuvas em excesso, seca, muito calor ou frio. O cultivo em ambientes protegidos (estufas) permite algum grau de controle sobre variáveis climáticas, principalmente luminosidade, temperatura, umidade do ar e intensidade do vento [Silva et al. 2014], o que pode levar a um aumento da produtividade. O uso de cultivo protegido aumentou rapidamente nas décadas de 1990 e 2000, e o Brasil se destaca na América do Sul por possuir a maior área com estufas [Silva et al. 2014]. A tendência é que este aumento se mantenha, impulsionado, principalmente, pela crescente demanda de alimentos de países com grande população, como a China [Hamshere et al. 2014] e as mudanças climáticas decorrentes do aquecimento global [Harvey 2000].

Dentre os principais usos de estufa no Brasil, se destaca o cultivo de hortaliças [Silva et al. 2014]. O uso de estufas para cultivo de hortaliças tem como principais benefícios o aumento de qualidade dos produtos, aumento da produtividade e, consequentemente, um aumento na rentabilidade para os agricultores [Reisser e Reichert 1998]. Entretanto, não basta acondicionar o cultivo em uma estufa para obter estes resultados, é preciso realizar o manejo adequado [Silva et al. 2014]. Com o manejo apropriado, é possível proporcionar condições favoráveis (temperatura, umidade, irrigação etc.) continuamente, para desenvolvimento das plantas, favorecendo a produtividade, e, simultaneamente, economizar água e energia [EMBRAPA 2012]. Considerando os aspectos mencionados, podemos argumentar que, quanto mais preciso for o controle da estufa, maior será o benefício proporcionado, pois as condições adequadas serão mantidas por mais tempo e eventuais problemas serão descobertos mais cedo. Desse modo, um dos caminhos interessantes e mais promissores que podemos seguir para possibilitar este controle mais apurado, e de forma remota, é o uso de tecnologia, principalmente nos casos em que as estufas estão em locais afastados e/ou de difícil acesso.

Diante das características supracitadas, se torna oportuno o estudo de abordagens baseadas no conceito de Internet of Things (IoT) [Xia et al. 2012], para construção de soluções que objetivam a automação/semi-automação de estufas. IoT é um conceito que abrange a comunicação entre dispositivos do cotidiano, como os aparelhos eletrônicos domésticos (geladeira, TV, microondas etc.) através de uma rede, que pode incluir a Internet. Este tipo de abordagem está dando origem a uma série de tecnologias, que promovem a comunicação de sistemas computacionais simples, como sensores, de maneira fácil e eficiente. O uso de tecnologias $I o T$ está criando muitas oportunidades para uma grande quantidade de novos aplicativos, que buscam melhorar a qualidade de nossas vidas.

Neste trabalho, propomos uma abordagem baseada em IoT de forma a gerenciar remotamente parâmetros do ecossistema interno de estufas, a fim de alcançar melhor qualidade no cultivo e maior produtividade. A arquitetura utilizada prevê o uso de sensores e atuadores, controlados por um sistema embarcado, que envia e recebe informações através de rede WI-FI para uma nuvem. Um dos diferenciais do trabalho é a utilização de um canal de contingência, para um caso de exceção no sistema. A solução também dispõe de um aplicativo móvel, que permite a visualização das condições da estufa pelo usuário.

\section{Fundamentação Teórica}

\subsection{MQTT}

O MQTT é um protocolo de mensageria que implementa o modelo de publisher e subscriber. Sua escolha se mostra apropriada ao projeto proposto devido características como: 
tamanho do cabeçalho e mensagens reduzidas, resultando em menor banda necessária para a comunicação, transmissão confiável das mensagens (personalizável em 3 níveis, at least once, at most once e exactly once). O próprio modelo publisher/subscriber com mensagens 1-N permite escalabilidade na distribuição de mensagens. Como abordagem escolhida, faz-se uso de um broker de MQTT na nuvem. Esse Broker funciona como intermediário, o Raspberry Pi atua como publisher e faz a publicação das mensagens com os dados dos sensores para o Broker. Sistemas interessados podem subscrever no tópico onde as mensagens são publicadas e o Broker se encarregará de enviar as mensagens para os subscritores.

\subsection{Raspberry Pi Zero W}

Uma das principais decisões do projeto foi utilizar o SOC Raspberry Pi Zero W (Rpi), que é um computador compacto de 32 bits, com uma ótima relação custo benefício para aplicações que não exigem um alto desempenho. O Rpi possui, internamente, um conjunto dispositivos capazes de trocar dados através barramentos internos, como por exemplo: ${ }^{2} \mathrm{C}$, SPI, WIFI, UART e pinos de saída programáveis, como entrada ou saída, Permitindo a conexão física direta com os sensores e atuadores necessários ao funcionamento do projeto. A interface WIFI, simplifica a sua comunicação com roteadores WI-FI e consequentemente o acesso à Internet.

\subsection{Sensor BME280}

O BME280 é um sensor de umidade desenvolvido especialmente para aplicações onde o tamanho e o baixo consumo de energia são características importantes. O componente combina sensores de alta linearidade e alta precisão e é viável para sistemas que necessitam de baixo consumo de corrente, estabilidade de longo prazo e alta robustez EMC (Compatibilidade Eletromagnética), conceito que permite diferentes dispositivos eletrônicos operarem sem interferência mútua. O sensor oferece um tempo de resposta muito rápido e, portanto, é adequado para satisfazer requisitos de desempenho para aplicações emergentes, como reconhecimento de contexto e alta precisão em uma ampla faixa de temperatura. A comunicação com o sensor pode ser realizada através de $\mathrm{I}^{2} \mathrm{C}$ [BOSCH 2021].

\subsection{Sensor MH-Z19b}

Para este projeto foi escolhido o sensor $\mathrm{MH}-\mathrm{Z19b}$ que usa o princípio não dispersivo infravermelho (NDIR) para detectar a existência de $\mathrm{CO}_{2}$ no ar. Esse tipo de sensor infravermelho de gás é desenvolvido pela integração afinada da tecnologia de detecção de gás absorvente infravermelho, com boa seletividade, não dependente de oxigênio e possui uma longa vida útil. Internamente faz a compensação de temperatura [Winsen, 2019]. O protocolo de comunicação deste sensor é UART. A latência do sensor apesar de grande, não é fator limitante da aplicação, pois a taxa de leitura dos sensores do sistema é de uma requisição a cada minuto. Dentre os sensores usados nesse trabalho, o MH-Z19b é o que possui o maior tempo de resposta.

\section{Abordagem Proposta}

A abordagem proposta neste trabalho (Figura 1) utiliza um conjunto de sensores para monitorar, principalmente, temperatura, umidade relativa e concentração de $\mathrm{CO}_{2}$ no ar. Os 
dados coletados por estes sensores são enviados para um software que é executado na SOC Rpi para o controle. Este software possui dois comportamentos principais: (i) tomar as ações necessárias através de relés, de acordo com parâmetros previamente estabelecidos, para controlar o ecossistema da estufa e (ii) enviar os dados para uma nuvem. Os dados disponibilizados na nuvem são consumidos por um aplicativo móvel, que permite ao usuário visualizar os parâmetros atuais do ecossistema da estufa (Figura 4). Atualmente, este aplicativo permite apenas a visualização das informações. Não é possível, a partir dele, enviar instruções para controle dos parâmetros da estufa. Como pode ser visto na Figura 1, existe um caminho alternativo para comunicação entre o software que roda na Rpi e o aplicativo móvel. Este caminho é utilizado apenas para situações de emergência, como falta de energia elétrica e aumento repentino da luminosidade, pois a mensagem chega mais rapidamente no usuário.

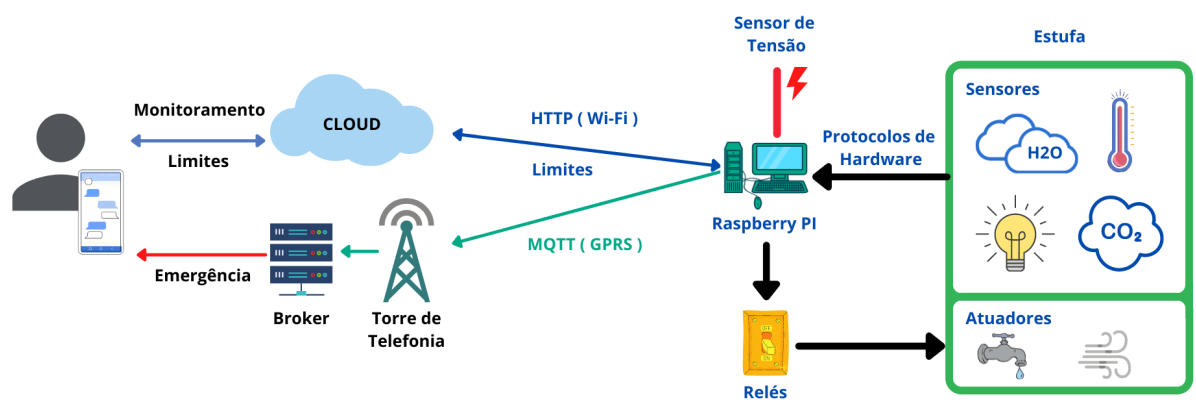

Figura 1. Interação entre as entidades do projeto

Fonte: Elaborado pelos autores do projeto

A figura 2 mostra como é feita a ligação física entre os componentes de sensoriamento (como o MH-Z19b para detectar concentração de $\mathrm{CO}_{2}$ ) e atuadores com a placa Raspberry (Rpi), através da UART.

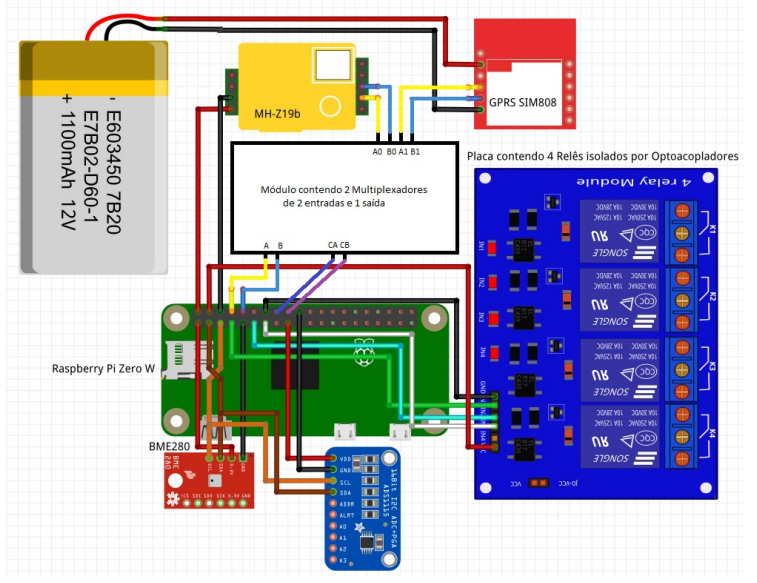

Figura 2. Ligação física entre os componentes da solução

Fonte: Elaborado pelos autores do projeto

A Rpi, não possui internamente um conversor analógico/digital. Assim, para interfacear com os sensores que são puramente analógicos, como: tensão, corrente e luminosidade, foi utilizado o conversor analógico digital ADS1115. Este sensor envia os dados digitalizados para Rpi através do protocolo $\mathrm{I}^{2} \mathrm{C}$. 


\subsection{Múltiplos de Canais de comunicação}

O uso de múltiplos canais de comunicação se faz necessário em caso de falhas no ecossistema, para envio de mensagens com urgência. Isso melhora a qualidade do serviço em geral, mitigando as chances de falhas catastróficas que ponham em risco o ecossistema da estufa. Nesta solução, utilizou-se dois canais distintos, um para envio de dados, um utilizando arquitetura cliente/servidor com protocolo HTTP e outro, para mensagens urgentes publish/subscriber com protocolo MQTT.

No paradigma cliente/servidor, o cliente é a própria unidade central que dá suporte aos sensores, localizada na própria estufa, enquanto o servidor é um serviço remoto, que tem por objetivo persistir informações em base de dados, por meio de interação programática controlada, ou seja, a base de dados é acessada por meio de padrões de comunicação e rotas estabelecidas pelo serviço remoto. A persistência é alcançada por meio da utilização de banco de dados relacional, que é componente integrante deste serviço remoto. Já na arquitetura publisher/subscriber a proposta é distribuir facilmente dados e informações para terceiros, os subscritores. Nesta solução, foi utilizado o protocolo de mensagens MQTT, por conta de suas características de funcionamento, como cabeçalhos de menor tamanho e mensagens curtas. Isso se faz importante uma vez que tais características são desejadas ao envio de mensagens a partir de dispositivos com recursos limitados, ou no caso de distribuição rápida de mensagens.

\subsection{Execução e Envio de Dados}

Como dito, a distribuição dos dados é feita por meio dos protocolos MQTT e HTTP. Esses serviços podem ser vistos na Figura 1. A proposta está organizada em componentes, configurações, serviços, variáveis de ambiente e arquivo de entrada. O primeiro são códigos que possuem métodos de utilidade geral, como por exemplo conversão de unidades de medida. Em seguida tem-se as configurações, onde as variáveis de ambiente são acessadas e disponibilizadas na forma de constantes globais. Os serviços são um conjunto de ferramentas que acessam os canais remotos, neste caso, o broker MQTT e a API web. Cada serviço foi separado por interesses, ou seja, cada classe tem um único conjunto de objetivos, correlacionados a um assunto. $\mathrm{O}$ arquivo principal é onde todas as entidades do projeto são instanciadas, tendo em vista os objetivos a serem alcançados. $\mathrm{O}$ funcionamento da aplicação segue a lógica apresentada na Figura 3.

\subsection{Software no RPi Zero W e Aplicativo Mobile}

A solução proposa neste trabalho é composta por uma placa de circuito integrado de custo reduzido, sensores capazes de realizar leituras cíclicas do ambiente como $\mathrm{CO}_{2}$, umidade e temperatura com o intuito de gravar os dados destas leituras em um servidor. Os dados podem então ser consultados pelo responsável da estufa através de uma aplicação mobile. O software responsável pela leitura dos dados consiste em um loop infinito estruturado na linguagem de programação Python, que oscila entre leitura e escrita de dados. A leitura dos dados é auxiliada pelas funções disponibilizadas nos apêndices da própria ficha técnica do BME280. O programa conta com o uso de bibliotecas como requests e JSON para a manipulação de requisições e JSON respectivamente.

Ao início de cada leitura, é realizada uma requisição ao servidor MySQL, com o intuito de recuperar os valores limite estipulados como máximos e mínimos de cada 


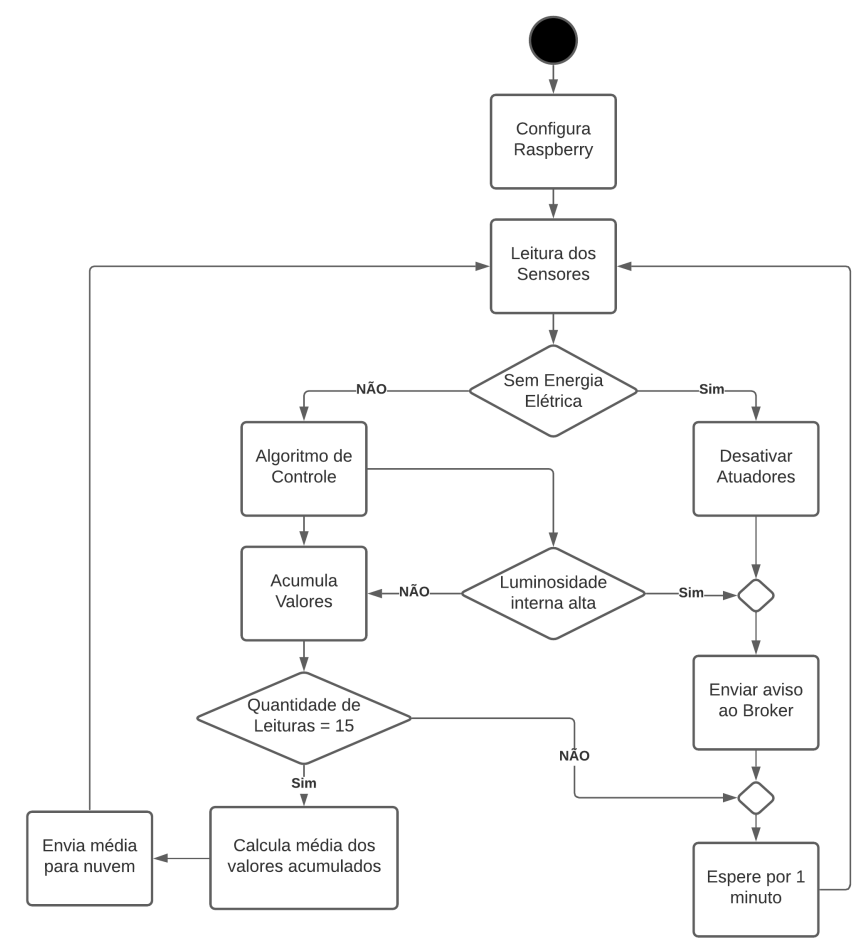

Figura 3. Diagrama de Atividades da Solução.

Fonte: Elaborado pelos autores do projeto

leitura. É baseado nesses valores recuperados que uma tratativa é feita para avaliar se os dados da leitura de um ciclo específico estão dentro dos padrões e, caso não estejam, é enviada uma notificação ao responsável da estufa. O erro também é guardado no banco de dados para consultas futuras e o dispositivo responsável por aquele dado é ligado ou desligado a fim de manter a estufa dentro dos parâmetros previamente estabelecidos.

Independente de estar dentro dos parâmetros, é realizada uma média móvel a cada 15 leituras com o intuito de reduzir "ruídos" presentes nos dados e, ao fim deste cálculo, o resultado é enviado ao banco de dados para consultas futuras. Já para a consulta de dados pelo cliente, os dados presentes no banco de dados são requisitados utilizando um aplicativo mobile baseado em React Native que tem três funcionalidades básicas: (i) a página principal contendo as últimas três notificações e três gráficos interativos criado a partir das leituras do dia; (ii) um painel de configurações que permite a visualização e alteração das variáveis que controlam os limites das leituras; e (iii) um log mais completo com todos os erros e notificações organizados pela data em que suas leituras foram realizadas. As páginas podem ser navegadas verticalmente de forma a exibir todas as informações caso não se adequem completamente ao visor do dispositivo móvel. A aplicação já se encontra desenvolvida e foi parcialmente testada.

\section{Conclusões}

Neste trabalho descrevemos uma infraestrutura para controle de estufas, com o objetivo de melhorar a qualidade e produtividade do cultivo. A abordagem utilizada inclui o uso de sensores para obtenção de dados do ecossistema da estufa. Um sistema computacional, composto de um software e uma Raspberry Pi Zero W, é responsável pela coleta dos 

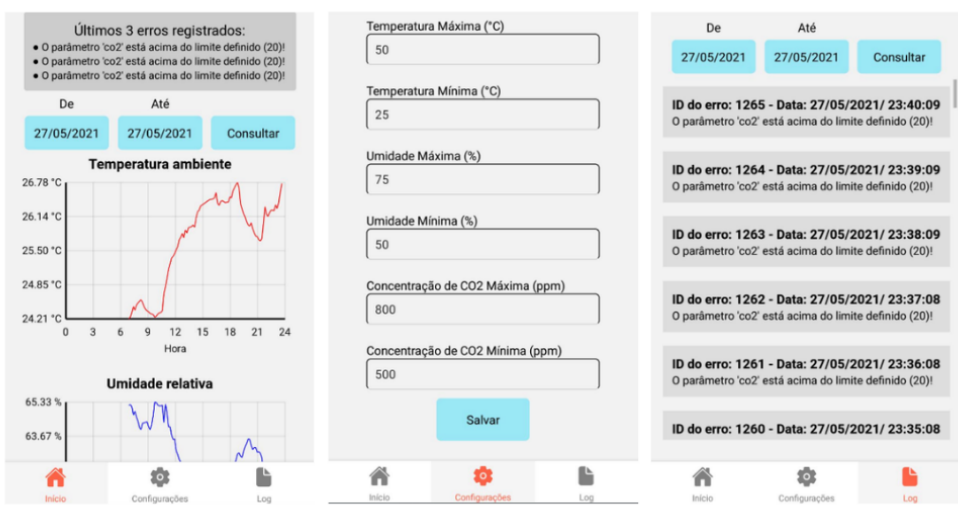

Figura 4. Interface mobile de monitoramento de temperatura e umidade.

Fonte: Elaborado pelos autores do projeto

dados dos sensores e sua transmissão para uma nuvem de dados. Este sistema também é responsável por acionar relés, para controlar as condições da estufa, caso estas não estejam de acordo com os parâmetros estabelecidos. Outro componente da infraestrutura é a nuvem, que armazena os dados através de um banco de dados relacional. Por fim, temos o aplicativo móvel, que acessa os dados da nuvem e possibilita a visualização das condições gerais do ecossistema da estufa pelo usuário. Uma característica importante da infraestrutura é a disponibilização de um canal independente, responsável por comunicações classificadas como urgente, o que trás uma maior robustez para a infraestrutura como um todo.

Existem diversas propostas na área de automação de estufas. Em [Litjens 2009] foi proposto um sistema baseado no protocolo ZigBee para monitorar temperatura e luminosidade. O módulo XBee foi utilizado para proporcionar economia de energia. Um estudo preliminar foi realizado para verificar a viabilidade de um sistema de baixo custo para controle de estufa em [Andrade 2017]. Foi criada uma estufa em escala, com os sensores e atuadores. Nenhuma infraestrutura de redes foi utilizada. Já em [Garcez et al.2018], uma abordagem teórica de sistema para controle de estufas é proposta. Nosso trabalho se destaca por oferecer uma interface através de aplicação móvel, facilitando a visualização das informações pelo usuário, e a disponibilização de um canal alternativo exclusivo para mensagens urgentes, o que permite uma comunicação mais célere com o usuário.

Os componentes do sistema proposto foram desenvolvidos e testados individualmente. Ainda não foram realizados testes em uma estufa real e este é um trabalho que pretendemos realizar no futuro, começando com avaliações através de uma estufa em escala. Além disto, pretendemos incrementar o aplicativo móvel, incorporando funcionalidades que permitam o controle das condições da estufa diretamente do celular. Neste caso, medidas de segurança robustas terão que ser adotadas, para impedir que terceiros 
acessem o sistema sem autorização e comprometam a estufa. Além disso, pretendemos incorporar outros elementos, como o uso do watchdog timer, para adicionar algum grau de tolerância a falhas na infraestrutura.

O presente trabalho foi realizado com apoio da FAPERJ.

\section{Referências}

CEPEA. Disponível em: https://www.cepea.esalq.usp.br/upload/ kceditor/files/Planilha_PIB_Cepea_Portugues_Site_2020.xlsx. Acesso em: Junho de 2020.

EMBRAPA. Manejo adequado da irrigação favorece a produtividade e a economia de água e energia. Disponível em: https://www.embrapa.br/busca-denoticias/-/noticia/1463432/manejo-adequado-da-irrigacaofavorece-a-produtividade-e-a-economia-de-agua-e-energia. Acesso em: 27 jun. 2021.

Raspberry Pi Foundation raspberry pi os. Disponível em: https:// www.raspberrypi.org. Acesso em: Junho 2020.

Andrade, P. H. G., Schreiber, L. A. L., Ceretta, E. G., and Rohde, T. M. (2017). Automação de estufas: Uma opção de baixo custo. Mostra Interativa da Produção Estudantil em Educação Científica e Tecnológica.

BOSCH Sensortech (2014). BME280 Combined humidity and pressure sensor. BOSCH Sensortech. Rev. 1.9.

Garcez, L. C., Miranda, E. A., De Oliveira, R., Silva, S. V., and Barcellos, R. G. (2018). Controle ambiental de instalações rurais utilizando microcontroladores. Exatas \& Engenharia, 8(22).

Hamshere, P., Sheng, Y., Moir, B., Syed, F., and Gunning-Trant, C. (2014). What china wants: Analysis of china's food demand to 2050. In ABARES (Australian Bureau of Agricultural and Resource Economics and Sciences) Conference Paper, volume 14.

Harvey, L. D. (2018). Global warming. Routledge.

Litjens, O. J. et al. (2009). Automação de estufas agrícolas utilizando sensoriamento remoto e o protocolo Zigbee. PhD thesis, UNIVERSIDADE DE SÃO PAULO.

Reisser Junior, C. and Reichert, L. (1998). Construcao de estufa modelo capela. Embrapa Clima Temperado-Documentos (INFOTECA-E).

Silva, B., Silva, A. d., and Pagiuca, L. (2014). Cultivo protegido: em busca de mais eficiência produtiva. Hortifruti Brasil, 1:10-18.

Winsen Electronics (2019). Infrared CO2 Sensor Module. Zhengzhou Winsen Electronics Technology Co., Ltd. Rev. 1.4.

Xia, F., Yang, L. T., Wang, L., and Vinel, A. (2012). Internet of things. International journal of communication systems, 25(9):1101. 